\title{
Fuzzy Logic Based Controller Design for Universal Motor Performance Enhancement
}

\author{
Shashank Shrivastava ${ }^{1}$, Imran Khan ${ }^{2}$, Mohd. Asif $\mathrm{Ali}^{3}$ \\ ${ }^{1}$ Azad Institute of Engineering and Technology, India, shashankshrivastava1992@gmail.com, \\ ${ }^{2}$ Azad Institute of Engineering and Technology, India, pe.imran@gmail.com, \\ ${ }^{3}$ Azad Institute of Engineering and Technology, India, asifaliec@ gmail.com,
}

\begin{abstract}
Universal motors are now a day's very versatile for applications which are dependent on force of fractional horsepower rating in almost all the domestic appliances related to the drilling, vacuum cleaners and mixers. Machine of such kind of jobs requires very high power/weight ratio is required to fulfill the need of high amount of torque at starting and the availability to run at direct current supply. Such type of appliances is also used as the motor starter for engines. These are series motor and excited by using winding connected in series to rotor winding and they are called universal motor because runs on both AC and DC supplies. In this work the results will be obtained on the PID controller design and fuzzy controller applied in an industrial motor control. It is a work that has been done to identify the strengths and weaknesses of each of the two drivers and dynamics under which each of them works.
\end{abstract}

Key words: Intelligent-Control, Analog Control, PID Controller, Fuzzy Logic, Expert Systems

\section{INTRODUCTION}

PID controllers are the most popular and commonly used for industrial process control and in its 60 years of existence are many applications that have been used, for example, in the temperature control of a boiler to control an AC motor. Despite being so used and known has always been the issue of the applicability of this driver in processes that are of nonlinear dynamics designs of motors as working under a PID controller is to control industrial processes is where enters involved the use of intelligent control with fuzzy logic [1].

Fuzzy logic is a mathematical fair where we have sought to respond to those problems where decision making is very similar to how human reasoning and fundamentals based on the experience we have of the systems. Its application in the areas of automatic control has been of great importance, especially in the process control system dynamics where it is non-linear order, for example the setting up of an inverted pendulum system, oscillatory systems and circuits LC mass or spring systems, etc.

On a commercial level is very common to find devices or everyday items which have been applied in control systems based on fuzzy logic is the common washing machine Fuzzy is said to improve cleaning clothes using fuzzy logic. Another application in which fuzzy logic can be found is in the throttle control and braking on subway type trains in Japan and with the use of fuzzy logic has achieved a smooth and efficient braking [4,5], similarly to boot. In the automotive industry using fuzzy logic has been a great impact because it has been given the responsibility of vehicle control systems such as brakes, suspension steering and engine. In the vehicular application has been made very significant progress since has increased the efficiency of fuel use and engine power of the vehicles, optimized brake system and preventing slippage during braking.

\section{FUZZY CONTROLLER}

A Fuzzy controller uses consistent control laws IF ... THEN logic rules in conjunction with fuzzy membership functions to control the process and minimize the error. The sets and fuzzy operators are the subjects and predicates of fuzzy logic. The logical rules are used to formulate If Then conditional expressions using fuzzy logic [2]. A fuzzy controller consists of four elements: the fuzzification, the defuzzification, the inference engine and the rule base.

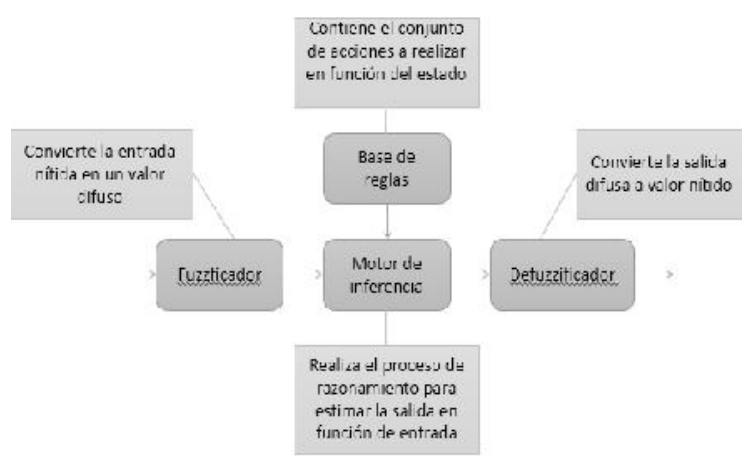

Figure 1: Fuzzy Logic system design

Inference Engine [3]: The cornerstone of any expert controller is its inference engine consisting of a set of expert rules, which reflect the knowledge base and the reasoning structure of the solution of any problem. A fuzzy control system (expert) is no exception and the rule base is the heart of nonlinear fuzzy controller shows in figure 1. 


\section{RELATED WORK}

Wireless strength transfer (WPT) the use of magnetic resonance is the era that could set human free from the traumatic wires. In reality [11], the WPT adopts the identical primary concept which has already been developed for at least 30 years with the time period inductive power transfer. WPT generation is growing rapidly in current years. At kilowatts strength stage, the transfer distance will increase from several millimeters to numerous hundred millimeters with a grid to load efficiency above $90 \%$. The advances make the WPT very attractive to the electrical vehicle (EV) charging packages in each stationary and dynamic charging eventualities. Siqi Li, (2015) [6] reviewed the technology within the WPT vicinity relevant to EV wi-fi charging. By introducing WPT in EVs, the obstacles of charging time, range, and value can be effortlessly mitigated. Battery technology is now not applicable in the mass marketplace penetration of EVs. It is was hoping that researchers will be recommended by means of the cuttingedge achievements, and push forward the further development of WPT as well as the enlargement of EV.

According to Botao Miao,(2016) [7] an equipment accommodates a power converter circuit and a controller. The electricity converter circuit consists of an inductor, a switching circuit, and a virtual manage loop circuit having an adjustable transfer feature, wherein the transfer function includes a zero variable and a sign benefit variable. The controller includes a tuning module configured to set a value for the 0 variable [12], set the sign benefit variable to a first benefit price, decide a manipulate mistakes for the first advantage price placing, in which the manipulate errors is a difference between a reference contemporary and a load present day at a circuit load, iteratively replace the benefit cost of the sign gain variable and decide the manage error for the up to date gain cost, and set an running benefit cost of the sign benefit variable to the advantage cost corresponding to a minimum manipulate blunders.

Bulky electrolytic capacitor is generally wished in bridgeless electricity issue correction rectifiers to buffer the double-frequency ripple strength (DFRP). However, it reduces the device reliability and power density considerably. Yonglu Liu, (2017)[8] proposed a control approach to divert DFRP to the small electricity switch capacitor. Then, the bulky electrolytic capacitor can be replaced with a small film capacitor. The proposed method is found out with the aid of making the first-rate of the prevailing switching states. Therefore, it needs no extra switches or power garage components, which might be normally required in different lively power decoupling techniques. The running precept is explained [13], and a closed-loop manipulate method is proposed. Finally, the effectiveness is confirmed through experimental results. In this letter, a new manipulate approach was proposed for bridgeless Cuk/Sepic PFC rectifier to reduce the output clear out capacitance. Then, the energy density and device reliability were stepped forward.
Conventional single-phase energy-component-correction (PFC) rectifiers with energetic strength decoupling capability normally require extra than 3 energetic switches in their circuits [14]. By exploring the concept of strengthbuffer mobile, a brand new single-level PFC rectifier with two active switches, one inductor and one small electricitybuffering capacitor is reported on this paper. Sinan Li, (2017) [9] proposed converter can attain high-strength factor, huge output voltage variety, and energy decoupling function without the usage of electrolytic capacitor. Additionally, an automatic strength decoupling manipulate scheme that is straightforward and clean to enforce is proposed in this paper. The running principle, manage technique, and design considerations of the proposed rectifier also are furnished. A one hundred-W prototype with a center voltage of a hundred and ten Vrms and a regulated dc output voltage ranging from 30 to a hundred $\mathrm{V}$ has been correctly designed and nearly examined.

Existing manage schemes for unmarried-phase ac-to-dc converters with lively power-decoupling characteristic commonly contain a committed strength-decoupling controller. Due to the exceptionally coupled and nonlinear nature of the unmarried-phase system, the layout of the power-decoupling controller (commonly primarily based on the small-signal linear manage strategies) is cumbersome, and the manage structure is complex. Additionally, with the prevailing energy-decoupling control, it's miles hard to acquire satisfied dynamic responses and strong circuit operation. Following a lately proposed automatic-power decoupling control scheme, this paper proposes a nonlinear control approach that can attain better huge-sign dynamic responses with strong disturbance rejection capability without the need for a devoted energy-decoupling controller. Sinan Li,(2018) [10] proposed controller has a easy shape, of which the layout is straightforward. The control approach can be without difficulty extended to different single-phase ac to dc systems with lively electricity-decoupling function. Simulation and experimental consequences validate the feasibility of the proposed control method on a -transfer dollar-enhance PFC rectifier prototype .

\section{PROPOSED WORK AND RESULTS}

To develop the project is meant to show results obtained through the design and simulation of a PID controller and a controller "fuzzy" in order that learn more about the design of controllers and determine the main characteristics of intelligent control with respect to a conventional control. The following shows the overall system diagram in figure 2 .

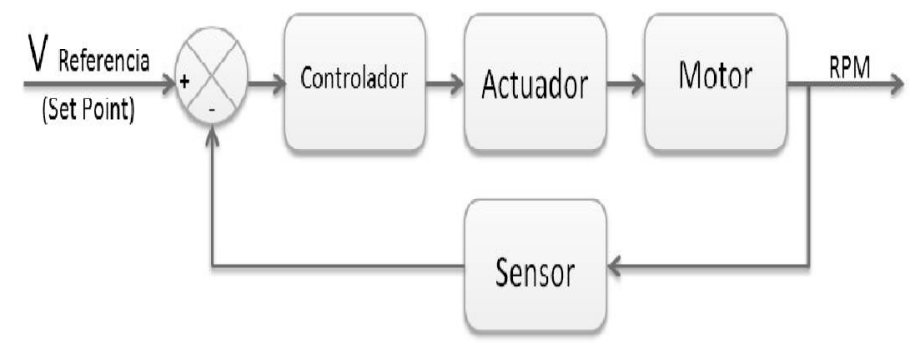

Figure 2:Block diagram of overall system 
During the development of the possible model on MATLAB, we have focused on the design of fuzzy controller and PID controllers that are simple and the goal is the analysis of results.

The plant or universal motor is given by the transfer function (1), this was taken from a real system, where there was an implementation and tests were performed concerning a conventional controller, for more information see [1]

$$
G(s)=3.839 \frac{1}{0.0304 s^{2}+0.387 s+1}
$$

To begin with the PID controller design, the first you have to observe and analyze the behavior of the closed loop system and know the system and define design parameters for proceeding with the implementation of control. We can observe the behavior closed-loop system to a unit step.

To design the fuzzy controller is taken into account multiple different approaches based on different sets of rules and to show that these controllers can be done in many ways and that some solutions may be better than others also can be seen that the definition of more or less sets significantly affect system performance [15].

In results we can see the system steady state error Ess ,settling time $\mathrm{Ts}$ and maximum overshoot $\mathrm{Mp}$ at the controller output, as the comparison between the system with fuzzy controller and PID. Then in the system will be evaluated before a amplitude perturbation in the system where [16] both the conventional controller and the intelligent control system reacts correctly to disturbance, this indicates that both controllers are efficient. Furthermore tests were also carried out with different steps shows in figure 3.

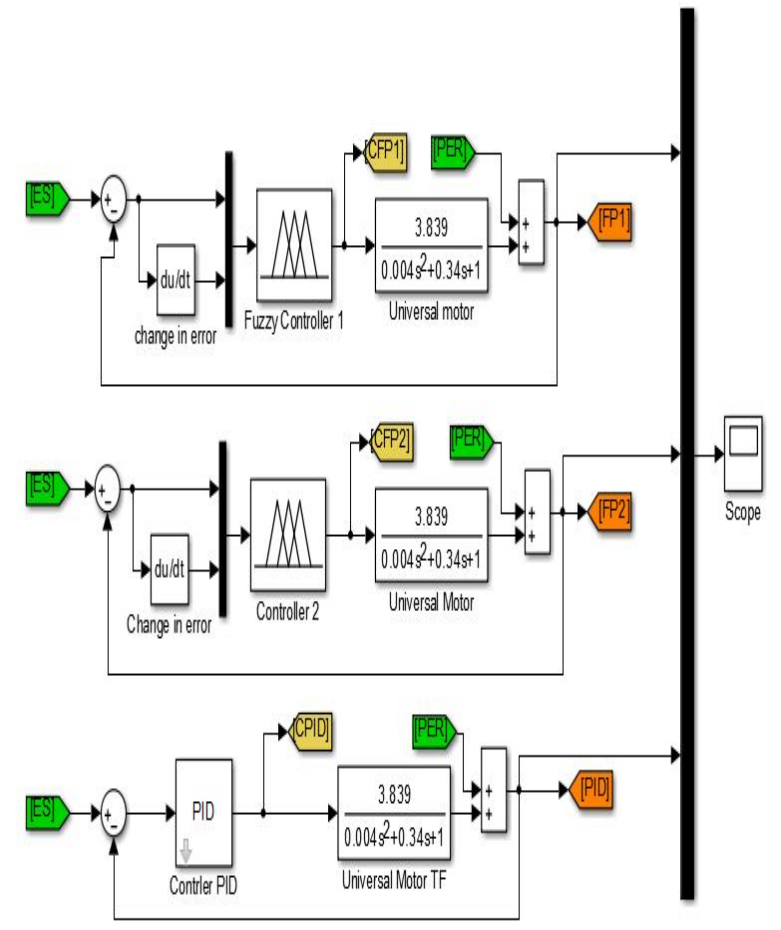

Figure 3: Block diagram of model developed in Simulink
The universal motor model transfer function is excited by step input along with the shift in the output after the steady state condition the response of all the three systems (PID, fuzzy controller 1,fuzzy controller 2) is observed and shown in figure 4. Time response using different controller shown in figure 5.

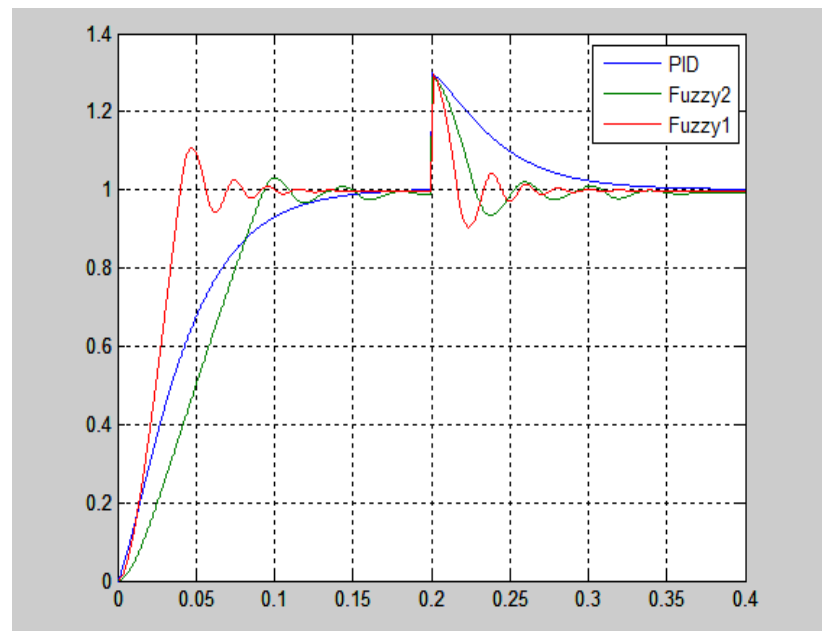

Figure 4: System time response using different controllers with shift in output values

Table 1: Performance of controller on universal motor

\begin{tabular}{|c|c|c|c|}
\hline $\begin{array}{c}\text { response } \\
\text { Performance } \\
\text { parameter }\end{array}$ & PID & Fuzzy 2 & Fuzzy1 \\
\hline Rise Time: & 0.0812 & 0.0672 & 0.0278 \\
\hline Settling Time: & 0.3056 & 0.2648 & 0.2527 \\
\hline Settling Min: & 0.9003 & 0.8937 & 0.8995 \\
\hline Settling Max: & 1.3003 & 1.2870 & 1.2977 \\
\hline Overshoot: & 29.9979 & 29.6737 & 29.9774 \\
\hline Undershoot: & 0 & 0 & 0 \\
\hline Peak: & 1.3003 & 1.2870 & 1.2977 \\
\hline Peak Time: & 0.2000 & 0.2000 & 0.2000 \\
\hline
\end{tabular}

Performance of controller on universal motor response is shown in table 1.

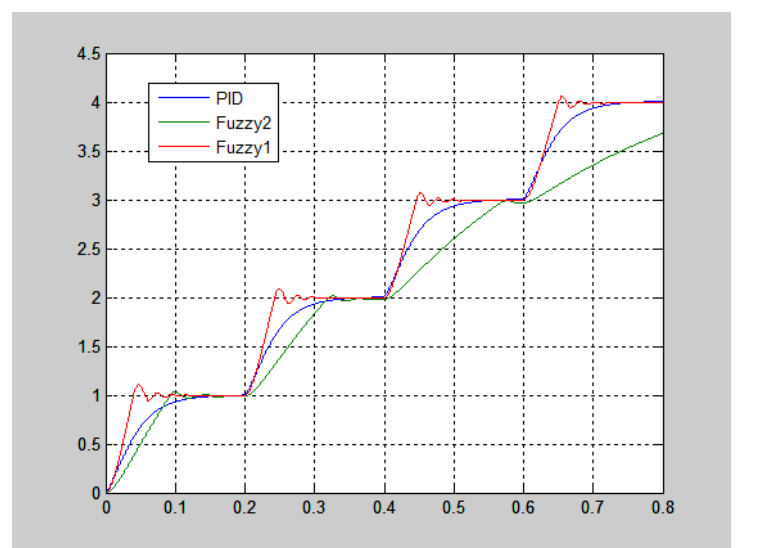

Figure 5: Time response using different controller at time varying step inputs values 


\section{CONCLUSION}

From the performance parameter shown in results it is concluded that the fuzzy logic controller is much better in some of the performance including rise time, settling time and robustness to system and input dynamics as compared to PID controller. The PID controller response for motor speed control based on tuning process is compared with Fuzzy controller. After evaluating the performance of Fuzzy controller, it is concluded that this controller gives quicker response than the PID controller. The parameters of FLC controller must be chosen and then retuning by using trial and error to get optimal value of parameters. The error signal in identification process can be depends as the cost function of optimally parameters value. Since FLC has advantages in fast response controlled with higher accuracy. But there are similar overshoot in system response for all the controller system.

\section{REFERENCES}

[1] John A. Diaz, Freddy E. Muñoz, "Design and Implementation of a Pid Controller Analog To A Universal Motor", BSD license, ID \# 38705, available at the website <http://www.mathworks.com/matlabcentral/fileexchange/38 705-control- universal motor pid-> [cited October 22, 2012]

[2] S. Y. Lee, S. Bhattacharya, T. Lejonberg, A. Hammad and S. Lefebvre, "Detailed Modeling of Static Var Compensators Using the Electromagnetic Transients Program (EMTP)", IEEE Trans. on Power Delivery, Vol. 7, No. 2, pp. 836-47, April 1992.

[3] Ali Zilouchian and Mohammad Jamshidi, "Intelligent Control Systems Using Soft Computing Methodologies", CRC, ISBN0-8493-875-0, 2001. https://doi.org/10.1201/9781420058147

[4] Kevin M,. "Passino And Stephen Yurkovich, Fuzzy Control", Addison Wesley, ISBN 0-201-18074-X, 1998.

[5] Yong-Won Cho, "Single Power-Conversion AC-DC Converter With High Power Factor and High Efficiency," IEEE transactions on power electronics, VOL. 29, NO. 9, september 2014

[6] Siqi Li, "Wireless Power Transfer for Electric Vehicle Applications," IEEE Journal of emerging and selected topics in power electronics, vol. 3, no. 1, march 2015

[7] Botao Miao, "Auto-Tuning Current Loop Compensation For Power Factor Correction Controller," U.S. Patent Jan. 5, 2016 US 9.231.469 B2

[8] Yonglu Liu, "A Control Method For Bridgeless Cuk/Sepic Pfc Rectifier To Achieve Power Decoupling," Ieee Transactions On Industrial Electronics, VOL. 64, NO. 9, SEPTEMBER 2017

https://doi.org/10.1109/TIE.2017.2688979

[9] Sinan Li, "A Single-Stage Two-Switch PFC Rectifier With Wide Output Voltage Range and Automatic AC Ripple Power Decoupling," IEEE Transactions On Power Electronics, VOL. 32, NO. 9, SEPTEMBER 2017

[10] Sinan Li, "Enhanced Automatic-Power-Decoupling Control Method for Single-Phase AC-to-DC Converters," IEEE Transactions On Power Electronics, VOL. 33, NO. 2, FEBRUARY 2018.
[11] Anurag, R. Sharma, " Load Forecasting by using ANFIS", International Journal of Research and Development in Applied Science and Engineering, Volume 20, Issue 1, 2020.

[12] R. Sharma, Anurag, " Load Forecasting using ANFIS A Review", International Journal of Research and Development in Applied Science and Engineering, Volume 20, Issue 1, 2020.

[13] R. Sharma, Anurag, " Detect Skin Defects by Modern Image Segmentation Approach, Volume 20, Issue 1, 2020.

[14] Anurag, R. Sharma, " Modern Trends on Image Segmentation for Data Analysis- A Review", International Journal of Research and Development in Applied Science and Engineering, Volume 20, Issue 1, 2020.

[15] Young Soo Jang et. al., "Development of the costeffective, miniaturized vein imaging system with enhanced noise reduction", International Journal of Advanced Trends in Computer Science and Engineering, Volume 8, No.6, November - December 2019.

https://doi.org/10.30534/ijatcse/2019/80862019

[16] Irma T. Plata1, et. al., "Development and Testing of Embedded System for Smart Detection and Recognition of Witches' Broom Disease on Cassava Plants using Enhanced Viola-Jones and Template Matching Algorithm", International Journal of Advanced Trends in Computer Science and Engineering, Volume 8, No.6, Volume 8, No.5, September - October 2019. 